\title{
Growth trajectory and pubertal tempo from birth till final height in a girl with Wolf-Hirschhorn syndrome
}

\author{
Jia Xuan Siew ${ }^{1}$ and Fabian Yap ${ }^{2}$ \\ 'Paediatric Medicine and 2Paediatric Endocrinology, KK Women's and Children's Hospital, Singapore, Singapore \\ Correspondence \\ should be addressed \\ to $\mathrm{J}$ S Siew \\ Email \\ siew.jia.xuan@singhealth. \\ com.sg
}

\section{Summary}

Growth anomaly is a prominent feature in Wolf-Hirschhorn syndrome (WHS), a rare congenital disorder caused by variable deletion of chromosome $4 \mathrm{p}$. While growth charts have been developed for WHS patients $0-4$ years of age and growth data available for Japanese WHS patients 0-17 years, information on pubertal growth and final height among WHS children remain lacking. Growth hormone (GH) therapy has been reported in two GH-sufficient children with WHS, allowing for pre-puberty catch up growth; however, pubertal growth and final height information was also unavailable. We describe the complete growth journey of a GH-sufficient girl with WHS from birth until final height (FH), in relation to her mid parental height (MPH) and target range (TR). Her growth trajectory and pubertal changes during childhood, when she was treated with growth hormone (GH) from 3 years 8 months old till 6 months post-menarche at age 11 years was fully detailed.

\section{Learning points:}

- Pubertal growth characteristics and FH information in WHS is lacking.

- While pre-pubertal growth may be improved by GH, GH therapy may not translate to improvement in FH in WHS patients.

- Longitudinal growth, puberty and FH data of more WHS patients may improve the understanding of growth in its various phases (infancy/childhood/puberty).

\section{Background}

Wolf-Hirschhorn syndrome $(1,2,3,4)$ (WHS) is a rare congenital disorder caused by variable deletion of the short arm of chromosome $4(2,5)$. Considered a contiguous gene syndrome (4), multiple phenotypic features of variable degree have been described in individuals depending on the extent of deletion. These include developmental delay, skeletal anomalies, hearing loss, cardiac, neurological and urinary tract abnormalities.

Growth anomalies are present in 80\% of affected individual (4) and include prenatal-onset growth failure, short stature and poor weight gain, although normal stature is possible in mild phenotypes. To depict growth patterns from 0 to 4 years of age, growth charts were developed using data from 101 patients from Netherlands, Great Britain, North America, Germany and Australia (6). Among Asians, growth of 34 WHS Japanese children 0-17 years were collated and plotted against local norms (7). Both data sets, as well as data from other case reports $(8,9)$, describe marked growth retardation among children with WHS. However, information on pubertal growth and final height (FH) among WHS children was lacking. Growth hormone (GH) therapy was used in two 
GH-sufficient children with WHS, allowing pre-puberty catch up growth (9); however, pubertal growth and FH information was unavailable.

We describe the complete growth journey of a girl with WHS from birth until $\mathrm{FH}$, in relation to her mid parental height (MPH) and target range (TR). We also describe her growth trajectory and pubertal changes during childhood, when she was treated with GH from 3 years 8 months old till 6 months post menarche at age 11 years. The advantage of our report is the detailed capture of all important information to characterize the growth and pubertal changes, until FH.

\section{Case presentation}

Our patient was born at 29-week gestation with birth weight of $880 \mathrm{~g}$, length $33 \mathrm{~cm}$ and $\mathrm{OFC} 23 \mathrm{~cm}$, which correspond to the 16th $(-0.99 \mathrm{sds}), 5$ th $(-1.64 \mathrm{sds})$ and 2nd (-2.13 sds) percentiles on the Fenton 2013 charts, respectively. Her parents were non-consanguineous with $\mathrm{MPH}$ at the 3-10th CDC percentile ( $-1.36 \mathrm{sds})$. There was history of premature rupture of membranes at 25-week gestation, for which IM dexamethasone was given. Her postnatal course was complicated by respiratory distress requiring intubation and surfactant, apnea of prematurity, presumed sepsis, bilateral grade 2 IVH, atrial septal defect (ASD), retinopathy of prematurity and poor weight gain requiring total parental nutrition and fortification of feeds. She was discharged home after 3 months of intensive neonatal care at corrected age of 40 weeks, when she weighed $2320 \mathrm{~g}$ and measured $46 \mathrm{~cm}$, which were 1st $(-2.55 \mathrm{sds})$ and $3 \mathrm{rd}(-1.95 \mathrm{sds})$ percentiles, respectively.

She continued to have developmental assessments and immunizations in the community setting after discharge, where she was noted to have motor (walked at age 2 years) and speech delay, as well as poor appetite and food refusal, such that she had weight faltering and height stunting. When first seen in the endocrine clinic at chronological age 2 years 4 months, she measured $7.01 \mathrm{~kg}$ and $76 \mathrm{~cm}$, giving BMI of $12.1 \mathrm{~kg} / \mathrm{m}^{2}$, which correspond to $-6.97 \mathrm{sds},-3.39 \mathrm{sds}$ and $-4.26 \mathrm{sds}$ on the CDC 2- to 20 -year growth charts (Fig. 1), respectively. Examination showed a mentally alert toddler, who was thin but did not appear wasted. She walked with support, and neurological, respiratory and abdomen examinations were normal. She received nutrition advice, and measured $10 \mathrm{~kg}, 83.3 \mathrm{~cm}$ and $14.4 \mathrm{~kg} / \mathrm{m}^{2}$ by the time she was 3 years 8 months or -4.13 sds, -3.81 sds and $-0.96 \mathrm{sds}$, respectively.

\section{Investigation}

Clinical diagnosis was not made at first presentation as she did not have classical features of WHS; however, she was dysmorphic (triangular face, beaked nose, frontal bossing), which prompted chromosome analysis that revealed $46 \mathrm{XX}$ with suspected $4 \mathrm{p}$ telomeric deletion. Deletion of the $4 \mathrm{p}$ region was confirmed by fluorescent in situ hybridization (FISH) using a probe for WolfHirschhorn Critical Region (WHSCR) within 4p16.3. Parental karyotyping was declined.

GH stimulation (IM Glucagon $1 \mathrm{mg}$ ) showed peak GH of 52 U/L (normal >20) and baseline IGF1 of $199 \mu \mathrm{g} / \mathrm{L}$ (51-218), excluding GH deficiency. Thyroid function was normal. Investigations also identified small kidneys for age and mild proteinuria, but normal renal function during annual assessment. At age 7 years, she underwent Amplatzer device closure of the persistent ASD.

\section{Treatment}

GH commenced $(0.03 \mathrm{mg} / \mathrm{kg} /$ day $)$ at 3 years 8 months on the basis of extreme short stature and poor height velocity despite improvement in BMI, and the first year response to $\mathrm{GH}$ was $8.6 \mathrm{~cm} /$ year, twice the pre-GH velocity of $4.1 \mathrm{~cm} /$ year (Figs 1 and 2). She was regular with GH injections (0.03-0.055 mg/kg/day), and there were no complications. She experienced feeding challenges and intermittent poor weight gain throughout her growing years but BMI remained age appropriate.

\section{Outcome and follow-up}

The GV range on GH before onset of secondary sex characteristics was $5-8.6 \mathrm{~cm} /$ year (from 4 years 8 months to 8 years 4 months). The pubertal GV pattern began after first clinical sign of adrenarche (i.e. onset of body odor) was observed at age 8 years 3 months, thelarche commenced from 9 years 6 months, peak GV occurred at 10 years and $\mathrm{FH}$ was attained at 13 years. The total pubertal height growth from thelarche to $\mathrm{FH}$ was $12.5 \mathrm{~cm}$. Height and GV measurements, in relation to GH therapy and sex characteristics are presented in Table 1 .

She is independent in activities of daily living, attends school for vocational training and is currently on multi-disciplinary (endocrinology/genetics/nephrology) follow-up. Latest renal ultrasound shows normal-sized kidneys, and she has mild proteinuria but normal renal function. 


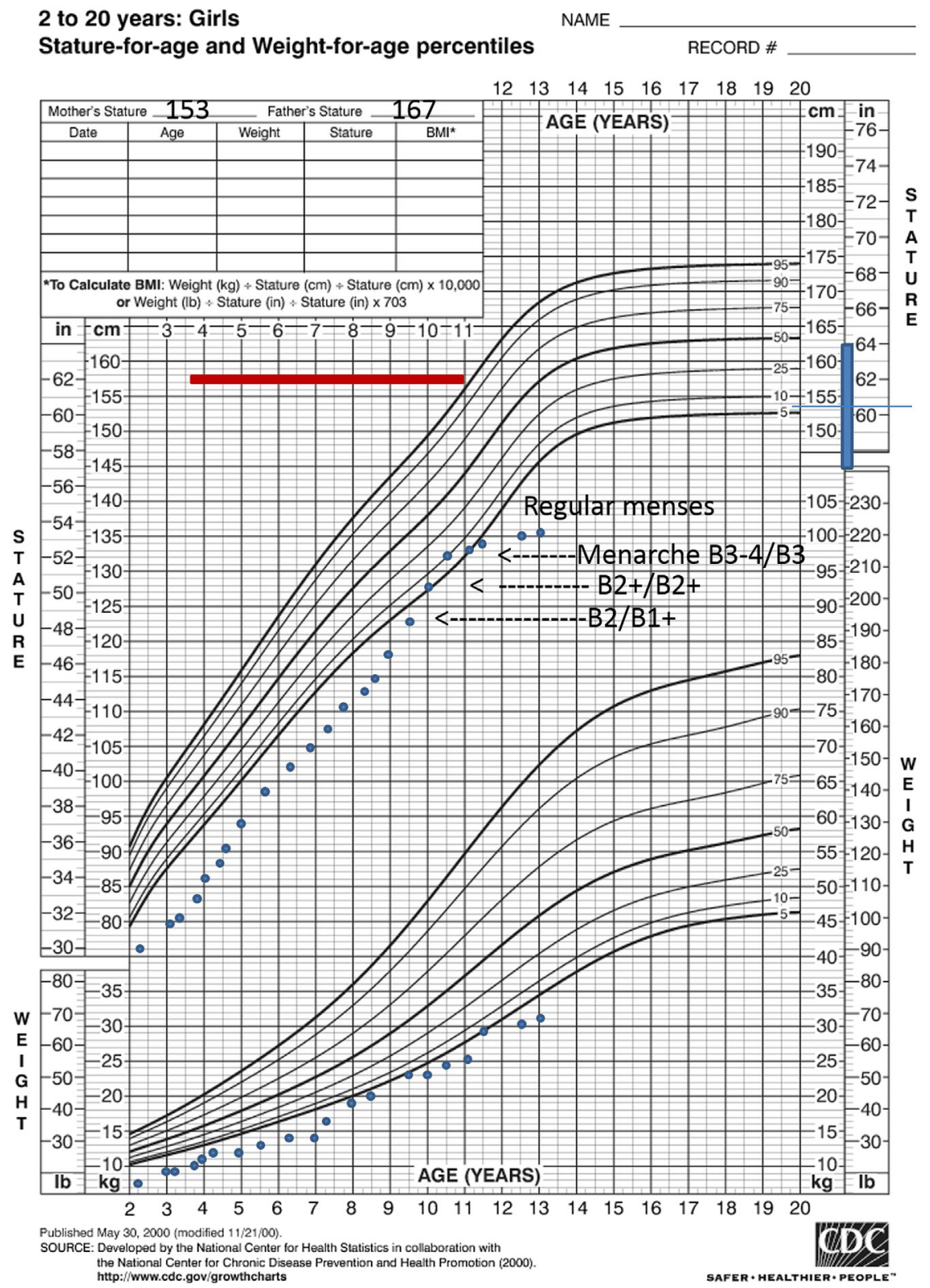

Figure 1

CDC 2000 2-20 years girl chart red horizontal bar represents duration of time on GH therapy, blue vertical bar represents the target range (TR) with the median representing mid parental height (MPH).

\section{Discussion}

We described the complete growth journey and pubertal characteristics of a girl with WHS until FH.

She presented at age 3 years with faltering growth. Premature and SGA at birth, she remained short at 40 weeks, failed to catch up in height at 2 years 4 months and faltered further at 3 years 8 months, in spite of normalization of BMI. Postnatal GV until 3 years 8 months was well below the population 3rd percentile. However, this height growth pattern was appropriate when plotted against WHS 0-4 years charts (6). Our patient was not GH deficient but commenced GH therapy. Although there is no widely accepted ethical or practice guidelines for GH in WHS, we made a clinical anthropometric decision to treat, in line with idiopathic short stature (ISS) criteria based on poor GV and extreme short stature (height at commencement was $83.3 \mathrm{~cm}$ (-3.81 sds), normal BMI 14.6 ( $-0.96 \mathrm{sds})$ and lack of growth standards for WHS children.

Like others (9), we found that GV approximated mean population GV when she was treated with GH 


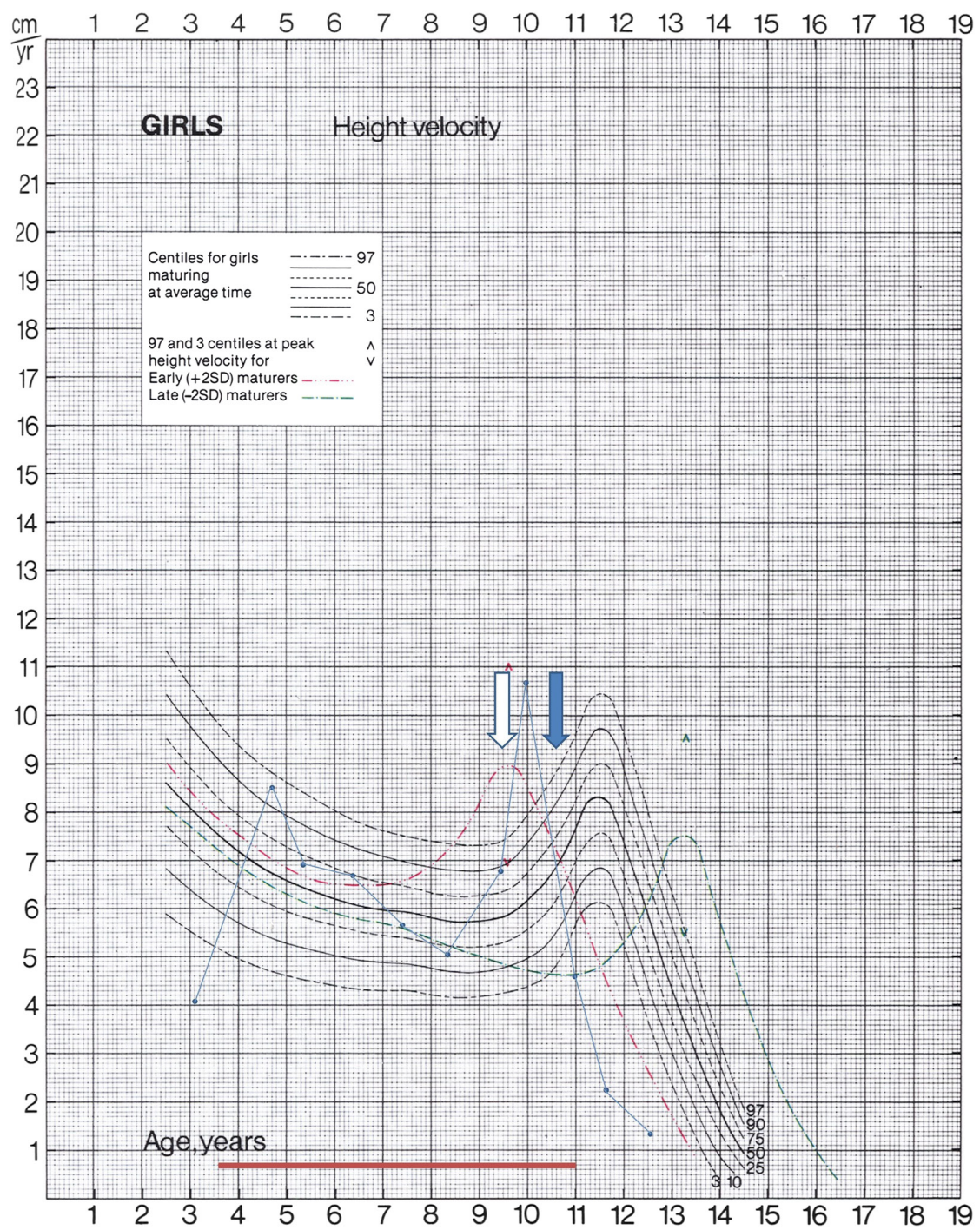

Figure 2

Height velocity girl chart red horizontal bar represents duration of time on GH therapy. White arrow represents thelarche and blue arrow represents menarche.

from 3 years 8 months till 8 years 8 months old (Fig. 2). However, as puberty and FH information of individuals with WHS are sparse, our report details the height and GV measurements in relation to the development of sex characteristics.

The age of onset of secondary sex characteristics were appropriate, with clinical adrenarche (body odor) starting at 8 years 3 months, thelarche at 9 years 6 months and pubarche at 10 years. As age of onset of puberty $(10,11,12)$ is defined by attainment of either Tanner stage 2 for breast or pubic hair, whichever earlier, the start of puberty in our patient was 9 years 6 months. This implies that the duration from thelarche to menarche was only 12 months, more rapid than usual compared to norms $(12,13,14,15)$. Rapid progression of puberty was proposed by Shimojima to be a unique pattern of growth in WHS girls that prevents sufficient duration for pubertal growth, resulting in inadequate pubertal height gain (7). By this definition of puberty, the total pubertal height gain of $12.5 \mathrm{~cm}$ seems severely compromised, compared to the estimated total pubertal growth of $19.2-21 \mathrm{~cm}$ in normal girls $(16,17)$.

The main advantage of our report relates to the detailed capture of key information to characterize the growth and pubertal changes, up till FH. Growth measurements were taken in triplicate by two consistent observers using a wall-mounted stadiometer and physical examination conducted in detail and documented. Notably, the family 
Table 1 Height and GV, in relation to GH therapy and sex characteristics.

\begin{tabular}{l}
\hline Age \\
\hline $3 y 8 m$ \\
$4 y 8 m$ \\
$8 y 3 m$ \\
$9 y 6 m$ \\
$10 y$ \\
$10 y 6 m$ \\
$11 y$ \\
$12 y 6 m$ \\
$13 y$
\end{tabular}

\begin{tabular}{l} 
Clinical characteristics \\
\hline Before GH \\
1y of GH \\
Adrenarche (body odour) \\
Thelarche (breast Tanner 2) \\
Pubarche (pubic hair Tanner 2) \\
Menarche, breast Tanner 3-4, axillary hair \\
GH stop, Bone age 13y 6m \\
Regular menses \\
Final height
\end{tabular}

was reliable, participative and duly attended all follow-up appointments.

In our patient, growth in the first 3 years of postnatal life was severely compromised, compounded by factors that include perinatal health, feeding challenges and the cardiac defect. These could have impacted FH through loss of early length/height growth and growth remained poor even after optimizing BMI from 2 years 4 months to 3 years 8 months. While pre-pubertal growth was improved by GH, GH therapy did not lead to improvement in FH, as previously postulated (9), in contrast to GH's proven impact on the FH of children with GH deficiency, Turner syndrome, SGA and ISS stature (18). Our patient had rapidly progressive puberty, and it is difficult to distinguish if this was due to WHS or normal genetic variation. Since there are limited reports of the pattern of puberty and $\mathrm{FH}$ of WHS patients, it remains unclear if WHS individuals as a whole are predisposed to rapidly progressive puberty. Should this be the case, we could then speculate whether the $4 \mathrm{p}$ region has a role in the regulation of the tempo of puberty. This is currently unknown.

We show pubertal take off (i.e. height acceleration) commence even before the onset of clinical thelarche or pubarche. We postulate that her underlying genetic condition may have contributed to the growth pattern via unknown mechanisms as she did not have malnutrition, significant functional renal or cardiac impairment or social challenges.

We acknowledge that our report is limited by lack of serial bone age data. This report cannot be used to generalize the growth of other WHS patients as this is not the natural history of growth of a WHS patient since there was the use of GH despite being tested GH sufficient. While it is difficult to draw firm conclusions from a single patient, GH therapy should not be expected to provide substantial long-term growth benefit to children with WHS. Review of longitudinal growth and puberty data of more WHS patients may improve the understanding of growth in its various phases (infancy/childhood/puberty)

\begin{tabular}{c}
\hline Height $(\mathrm{cm})$ \\
\hline 83.3 \\
91.9 \\
112.9 \\
122.5 \\
127.5 \\
132.1 \\
133 \\
135 \\
135
\end{tabular}

\begin{tabular}{c}
\hline Height velocity (cm/year) \\
\hline 4.1 \\
8.6 \\
5 (GV nadir) \\
6.8 \\
10.7 (GV peak) \\
- \\
4.5 \\
1.4 \\
$<1$
\end{tabular}

and FH. This may help in constructing growth charts for older children with WHS, similar to charts in more common genetic conditions like Down syndrome and Turner syndrome so that informed decisions may be made in other WHS patients considering GH therapy.

\section{Declaration of interest}

The authors declare that there is no conflict of interest that could be perceived as prejudicing the impartiality of the research reported.

\section{Funding}

This research did not receive any specific grant from any funding agency in the public, commercial or not-for-profit sector.

\section{Patient consent}

Written informed consent has been obtained from the patient's guardian for publication of the submitted article and accompanying images.

Author contribution statement

Jiaxuan Siew prepared the manuscript and participated in the clinical care of the patient. Fabian Yap critically reviewed and edited the manuscript and is the primary endocrinologist of the patient.

\section{References}

1 Battaglia A, Carey JC \& Wright TJ. Wolf-Hirschhorn (4p-) syndrome. Advances in Pediatrics 200148 75-113.

2 Battaglia A, Carey JC \& South ST. Wolf-Hirschhorn syndrome. In GeneReviews $^{\circledR}$. Eds MP Adam, HH Ardinger, RA Pagon, SE Wallace, LJH Bean, K Stephens \& A Amemiya. Seattle, WA: University of Washington, 2015.

3 Battaglia A, Carey JC \& South ST. Wolf-Hirschhorn syndrome: a review and update. American Journal of Medical Genetics Part C, Seminars in Medical Genetics 2015169 216-223. (https://doi.org/10.1002/ ajmg.c.31449)

4 Battaglia A, Filippi T \& Carey JC. Update on the clinical features and natural history of Wolf-Hirschhorn (4p-) syndrome: experience with 87 patients and recommendations for routine health supervision. American Journal of Medical Genetics Part C, Seminars in Medical Genetics 2008 148C 246-251. (https://doi.org/10.1002/ajmg.c.30187)

5 Sukarova-Angelovska E, Kocova M, Sabolich V, Palcevska S \& Angelkova N. Phenotypic variations in wolf-hirschhorn syndrome. 
Balkan Journal of Medical Genetics: BJMG 201417 23-30. (https://doi. org/10.2478/bjmg-2014-0021)

6 Antonius T, Draaisma J, Levtchenko E, Knoers N, Renier W \& van Ravenswaaij C. Growth charts for Wolf-Hirschhorn syndrome (0-4 years of age). European Journal of Pediatrics 2008167 807-810.

7 Shimojima K \& Yamamoto T. Growth profiles of 34 patients with Wolf-Hirschhorn Syndrome. Journal of Pediatric Genetics 20121 33-37. (https://doi.org/10.3233/PGE-2012-007)

8 Titomanlio L, Romano A, Conti A, Genesio R, Salerno M, De Brasi D, Nitsch L \& Del Giudice E. Mild Wolf-Hirschhorn phenotype and partial GH deficiency in a patient with a $4 \mathrm{p}$ terminal deletion. American Journal of Medical Genetics Part A 2004 127A 197-200. (https://doi.org/10.1002/ajmg.a.20667)

9 Austin DE, Gunn AJ \& Jefferies CA. Severe short stature and WolfHirschhorn syndrome: response to growth hormone in two cases without growth hormone deficiency. Oxford Medical Case Reports 20152015 211-214. (https://doi.org/10.1093/omcr/omv008)

10 Biro FM, Huang B, Daniels SR \& Lucky AW. Pubarche as well as thelarche may be a marker for the onset of puberty. Journal of Pediatric and Adolescent Gynecology 200821 323-328. (https://doi. org/10.1016/j.jpag.2007.09.008)

11 Ducharme JR \& Collu R. Pubertal development: normal, precocious and delayed. Clinical Endocrinology and Metabolism 198211 57-87.

12 Rosenfield RL, Lipton RB \& Drum ML. Thelarche, pubarche, and menarche attainment in children with normal and elevated body mass index. Pediatrics 2009123 84-88. (https://doi.org/10.1542/ peds.2008-0146)

13 German A, Shmoish M \& Hochberg Z. Predicting pubertal development by infantile and childhood height, BMI, and adiposity rebound. Pediatric Research 201578 445-450. (https://doi. org/10.1038/pr.2015.129)

14 Hui LL, Lam HS, Leung GM \& Schooling CM. Duration of puberty in preterm girls. American Journal of Human Biology 201729 e22963. (https://doi.org/10.1002/ajhb.22963)

15 Marti-Henneberg C \& Vizmanos B. The duration of puberty in girls is related to the timing of its onset. Journal of Pediatrics 1997131 618-621. (https://doi.org/10.1016/S00223476(97)70073-8)

16 Marshall WA \& Tanner JM. Variations in pattern of pubertal changes in girls. Archives of Disease in Childhood 196944 291-303. (https:// doi.org/10.1136/adc.44.235.291)

17 Buckler JM \& Wild J. Longitudinal study of height and weight at adolescence. Archives of Disease in Childhood 198762 1224-1232. (https://doi.org/10.1136/adc.62.12.1224)

18 Ranke MB \& Lindberg A. Observed and predicted total pubertal growth during treatment with growth hormone in adolescents with idiopathic growth hormone deficiency, Turner syndrome, short stature, born small for gestational age and idiopathic short stature: KIGS analysis and review. Hormone Research Paediatrics 201175 423-432. (https://doi.org/10.1159/000324117)

Received in final form 4March2018

Accepted 19March2018 